\title{
Phenomenology of the minimal B-L extension of the Standard Model
}

\author{
L. Basso ${ }^{* 1}$, A. Belyaev ${ }^{1}$, S. Moretti ${ }^{1,2}$, G.M. Pruna ${ }^{1}$ and \\ C.H. Shepherd-Themistocleous ${ }^{1, \dagger}$ \\ ${ }^{1}$ NExT Institute, University of Southampton, Highfield, Southampton, and RAL, Chilton, Didcot, \\ $U K$ \\ ${ }^{2}$ Dipartimento di Fisica Teorica, Università di Torino, Torino, Italy \\ E-mail: 1b4x07@soton.ac.uk, a.belyaev@soton.ac.uk, \\ s.moretti@soton.ac.uk, g.m.pruna@soton.ac.uk, claires@mail.cern.ch
}

\begin{abstract}
We present the Large Hadron Collider (LHC) discovery potential in the $Z^{\prime}$ and heavy neutrino sectors of a $U(1)_{B-L}$ enlarged Standard Model also encompassing three heavy Majorana neutrinos. This model exhibits novel signatures at the LHC, the most interesting arising from a $Z^{\prime}$ decay chain involving heavy neutrinos, eventually decaying into leptons and jets. In particular, this signature allows one to measure the $Z^{\prime}$ and heavy neutrino masses involved. In addition, over a large region of parameter space, the heavy neutrinos are rather long-lived particles producing distinctive displaced vertices that can be seen in the detectors. Lastly, the simultaneous measurement of both the heavy neutrino mass and decay length enables an estimate of the absolute mass of the parent light neutrino. For completeness, we will also compare the LHC and a future Linear Collider (LC) discovery potentials.
\end{abstract}

European Physical Society Europhysics Conference on High Energy Physics

July 16-22, 2009

Krakow, Poland

\footnotetext{
* Speaker.

${ }^{\dagger}$ This work is supported in part by the SEPnet Physics Consortium. SM is financially supported in part by the scheme 'Visiting Professor - Azione D - Atto Integrativo tra la Regione Piemonte e gli Atenei Piemontesi.
} 


\section{Introduction}

The $B-L$ (baryon number minus lepton number) symmetry plays an important role in various physics scenarios beyond the Standard Model (SM). First, the gauged $U(1)_{B-L}$ symmetry group is contained in several Grand Unified Theories. Second, the scale of the $B-L$ symmetry breaking is related to the mass scale of the heavy right-handed Majorana neutrino mass terms providing the well-known see-saw mechanism of light neutrino mass generation. Third, the $B-L$ symmetry and the scale of its breaking are tightly connected to the baryogenesis mechanism through leptogenesis via sphaleron interactions preserving $B-L$.

In this work, we study in detail the collider phenomenology of the gauge and fermionic sectors of the minimal $B-L$ extension of the SM, i.e., the $Z^{\prime}$ and the three heavy neutrinos and their interplay, in the framework of the LHC. A very interesting feature of such a $B-L$ model is possibly relatively long lifetimes of the heavy neutrinos, which can directly be measured. Such measurement could also be a key to shedding light on the mass spectra of the light neutrinos.

\section{The model}

The model under study is the so-called "pure" or "minimal" $B-L$ model (see [1] for conventions and references) since it has vanishing mixing between the two $U(1)_{Y}$ and $U(1)_{B-L}$ groups. In this model the classical gauge invariant Lagrangian, obeying the $S U(3)_{C} \times S U(2)_{L} \times U(1)_{Y} \times$ $U(1)_{B-L}$ gauge symmetry, can be decomposed as usual as $\mathscr{L}=\mathscr{L}_{Y M}+\mathscr{L}_{s}+\mathscr{L}_{f}+\mathscr{L}_{Y}$. The nonAbelian field strengths in $\mathscr{L}_{Y M}$ are the same as in the SM whereas the Abelian ones can easily be diagonalised ${ }^{1}$. In such field basis, the covariant derivative is: $D_{\mu} \equiv \partial_{\mu}+i g_{S} T^{\alpha} G_{\mu}{ }^{\alpha}+i g T^{a} W_{\mu}{ }^{a}+$ $i g_{1} Y B_{\mu}+i\left(\widetilde{g} Y+g_{1}^{\prime} Y_{B-L}\right) B_{\mu}^{\prime}$. The "pure" or "minimal" $B-L$ model is defined by the condition $\widetilde{g}=0$, that implies no mixing between the $Z^{\prime}$ and the SM $Z$ gauge bosons.

The fermionic Lagrangian is the same as in the SM, apart from the term associated to RHneutrinos ( $i \overline{v_{k R}} \gamma_{\mu} D^{\mu} v_{k R}$, where $k$ is the generation index). The fields' charges are the usual SM and $B-L$ ones (in particular, $B-L=1 / 3$ for quarks and -1 for leptons). The latter charge assignments as well as the introduction of three new fermionic right-handed heavy neutrinos $\left(v_{R}\right)$ and one scalar Higgs $(\chi$, charged +2 under $B-L)$ fields are designed to eliminate the triangular $B-L$ gauge anomalies and to ensure the gauge invariance of the theory, respectively. Therefore, the $B-L$ gauge extension of the SM group broken at the Electro-Weak (EW) scale does necessarily require at least one new scalar field and three new fermionic fields which are charged with respect to the $B-L$ group.

Regarding the scalar Lagrangian, the only differences with respect to the SM is that we have to introduce a kinetic term for the $\chi$ field as well as to modify the scalar potential, given by $V(H, \chi)=$ $m^{2} H^{\dagger} H+\mu^{2}|\chi|^{2}+\lambda_{1}\left(H^{\dagger} H\right)^{2}+\lambda_{2}|\chi|^{4}+\lambda_{3} H^{\dagger} H|\chi|^{2}$, where $H$ and $\chi$ are the complex scalar Higgs doublet and singlet fields, respectively.

Finally, as for Yukawa interactions, we are allowed to introduce two new terms $\left(y_{j k}^{v} \overline{l_{j L}} v_{k R}\right.$ $\widetilde{H}+y_{j k}^{M} \overline{\left(v_{R}\right)_{j}^{c}} v_{k R} \chi$, where $\widetilde{H}=i \sigma^{2} H^{*}$ and $i, j, k$ take the values 1 to 3 ), where the first term is the usual Dirac contribution and the second term is the Majorana one. Neutrino mass eigenstates,

\footnotetext{
${ }^{1}$ In general, Abelian field strengths tend to mix and the diagonalisation of the kinetic terms could be complicated. However, in our case just one off-diagonal term appears and a linear $2 \times 2$ transformation is sufficient to fulfill our aim.
} 
obtained after applying the see-saw mechanism, will be called $v_{l}$ and $v_{h}$, being the former the SM-like ones. With a reasonable choice of the Yukawa couplings, the heavy neutrinos $v_{h}$ can have masses $m_{v_{h}} \sim \mathscr{O}(100) \mathrm{GeV}$, within the LHC reach. Their role will be discussed later on.

\section{Discovery potential at the LHC and at future LC}

We want to explore the discovery potential of hadronic and leptonic machines in the $M_{Z^{\prime}} g_{1}^{\prime}$ plane of our model, in the di-muon production process. We compare the LHC hadronic scenario, with $100 \mathrm{fb}^{-1}$ data collected, to two different LC leptonic frameworks, at a fixed Centre-of-Mass (CM) energy of $\sqrt{s_{e^{+} e^{-}}}=3 \mathrm{TeV}$ (500 $\mathrm{fb}^{-1}$ data altogether) and in a so-called energy scan, where the CM energy is set to $\sqrt{s_{e^{+} e^{-}}}=M_{Z^{\prime}}+10 \mathrm{GeV}$ and we assume $10 \mathrm{fb}^{-1}$ of luminosity for each step. We then limit both signal and background to the detector acceptance volumes and $M_{\mu \mu}$ to an invariant mass window defined by the CMS and ILC prototype resolution [2] or $3 \Gamma_{Z^{\prime}}$, whichever the largest. We finally define the significance $\sigma$ as $s / \sqrt{b}$ ( $s$ and $b$ being the signal and background event rates, respectively $)^{2}$ : the discovery will be for $\sigma \geq 5$, as usual.

As a result, for $M_{Z^{\prime}}>800 \mathrm{GeV}$, the LC potential to explore the $M_{Z^{\prime}} g_{1}^{\prime}$ parameter space in the fixed CM energy approach goes beyond the LHC reach. For example, for $M_{Z^{\prime}}=1 \mathrm{TeV}$, the LHC can discover a $Z^{\prime}$ if $g^{\prime} \approx 0.007$ while a LC can achieve this for $g^{\prime} \approx 0.005$. The difference is even more drastic for larger $Z^{\prime}$ masses: a LC can discover a $Z^{\prime}$ with a $2 \mathrm{TeV}$ mass for a $g_{1}^{\prime}$ coupling which is a factor 8 smaller.

In case of the energy scan approach, the $M_{Z^{\prime}}-g_{1}^{\prime}$ parameter space can be probed even further for $M_{Z^{\prime}}<1.75 \mathrm{TeV}$. For example, for $M_{Z^{\prime}}=1 \mathrm{TeV}, g_{1}^{\prime}$ couplings can be probed down to the $2.6 \times 10^{-3}$, following a $Z^{\prime}$ discovery. Furthermore, the parameter space corresponding to the mass interval $500 \mathrm{GeV}<M_{Z^{\prime}}<1 \mathrm{TeV}$, which the LHC covers better as compared to a LC with fixed energy, can be accessed well beyond the $\mathrm{LHC}$ reach with a $\mathrm{LC}$ in energy scan regime.

\section{4. $Z^{\prime}$ and neutrino phenomenology}

The possibility of the $Z^{\prime}$ gauge boson decaying into pairs of heavy neutrinos is one of the most significant results of this work since, in addition to the clean SM-like di-lepton signature, it provides multi-lepton signatures where backgrounds can strongly be suppressed.

In order to address this quantitatively, we first determine the relevant Branching Ratios (BRs): clearly, these depend strongly on the heavy neutrino mass. A feature of the current $B-L$ model is that the $Z^{\prime}$ predominantly couples to leptons. In fact, after summing over the generations, we roughly get for leptons a total BR of $3 / 4$ and for quarks the remaining $1 / 4$. Not surprisingly then, for a relatively light (with respect to the $Z^{\prime}$ gauge boson) heavy neutrino, the $Z^{\prime}$ BR into pairs of such particles is relatively high: $\sim 20 \%$ (at most, after summing over the generations). Regarding finally the total $Z^{\prime}$ width, it strongly depends on the coupling up to few hundreds $\mathrm{GeV}$ for a $\mathrm{TeV}$ scale gauge boson.

Moving to the neutrino sector, after the see-saw diagonalisation of the neutrino mass matrix, we obtain three very light neutrinos $\left(v_{l}\right)$, which are the SM-like neutrinos, and three heavy neutrinos

\footnotetext{
${ }^{2}$ This definition, based on a gaussian distribution, is valid when the number of events is large enough, i.e. $s, b>20$. Otherwise, in case of lower statistics, we exploited the Bityukov algorithm [3], which uses the Poisson 'true' distribution.
} 
$\left(v_{h}\right)$. The latter have an extremely small mixing with the $v_{l}$ 's thereby providing very small but non-vanishing couplings to gauge and Higgs bosons. Hence, neglecting the scalar sector, the $v_{h}$ 's prefer to decay into SM gauge bosons, as well as into the new $Z^{\prime}$, when these decay channels are kinematically allowed. In details, $\operatorname{BR}\left(v_{h} \rightarrow l^{\mp} W^{ \pm}\right)$is dominant and reaches the $2 / 3$ level in the $M_{Z^{\prime}}>M_{v_{h}} \gg M_{W}, M_{Z}$ limit, while the $\mathrm{BR}\left(v_{h} \rightarrow v_{l} Z\right)$ represents the remaining $1 / 3$ in this regime.

The $v_{h}$ couplings to the gauge bosons are proportional to the ratio of light to heavy neutrino masses, which is extremely small. Therefore the decay width of the heavy neutrino is correspondingly small and its lifetime large: it can be a long lived particle and, over a large portion of parameter space, its lifetime can be comparable to or exceed that of the $b$-quark, giving rise to a displaced vertex inside the detector. The key point is that a measurable lifetime along with a mass determination for $v_{h}$ also enables a determination of $m_{v l}$, by just inverting the see-saw formula.

\section{Heavy neutrino mass measure at the LHC}

Multi-lepton signatures carry the hallmark of the heavy neutrinos as the latter enter directly the corresponding decay chains. We performed a detailed Monte Carlo analysis at the benchmark point $M_{Z}^{\prime}=1.5 \mathrm{TeV}, g_{1}^{\prime}=0.2$ and $M_{v_{h}}=200 \mathrm{GeV}$, with $\sigma\left(p p \rightarrow v_{h} v_{h}\right)=46.7 \mathrm{fb}$ (for CTEQ6L PDFs with $Q^{2}=M_{Z^{\prime}}^{2}$ ), and the decay we are interested in is $v_{h} v_{h} \rightarrow 3 l+2 q+v_{l}$, with a significant fraction of missing energy. A very suitable distribution to look at turned out to be the transverse mass defined in [4], i.e., $m_{T}^{2}=\left(\sqrt{M^{2}(v i s)+P_{T}^{2}(v i s)}+\left|P_{T}\right|\right)^{2}-\left(\vec{P}_{T}(v i s)+\vec{P}_{T}\right)^{2}$, where (vis) means the sum over the visible particles. If the visible particles we sum over are the 3 leptons and 2 jets, the transverse mass distribution will peak at the $Z^{\prime}$ mass. We can also see evidence for the presence of a heavy neutrino by just considering as visible particles the 2 leptons with the smallest azimuth-rapidity separation, since this is the topology relevant to a $v_{h}$ decay. The results show that this transverse mass peak for the heavy neutrino is likely to be the best way to measure its mass. The striking signature of this model is that both of the above peaks occur simultaneously.

It is important to note that the backgrounds are completely under control: as sources we considered $W Z j j, t \bar{t}$ (where a further lepton comes from a semileptonic $b$ decay) and $t \bar{t} l v$. After simple kinematic and detector acceptance requirements, significant suppression of the backgrounds comes from requiring the di-jet invariant mass to be close to the $W$ mass (as this is the signal topology). At this stage, just the $W Z j j$ is comparable to our signal, but the $Z^{\prime}$ peak described above can be used to further reduce the remnant background.

\section{References}

[1] L. Basso, A. Belyaev, S. Moretti and C. H. Shepherd-Themistocleous, arXiv:0812.4313 [hep-ph] (to appear in PRD); L. Basso, A. Belyaev, S. Moretti and G. M. Pruna, arXiv:0903.4777 [hep-ph].

[2] G. L. Bayatian et al. [CMS Collaboration], preprint CERN-LHCC-2006-001, CMS-TDR-008-1; T. Behnke et al. [ILC Collaboration], arXiv:0712.2356 [physics.ins-det].

[3] S. I. Bityukov and N. V. Krasnikov, Nucl. Instr. and Meth. A452 (2000) 518.

[4] V. Barger, T. Han and R.J.N. Phillips, Phys. Rev. D 36 (1987) 295. 